\title{
An Adaptive Detector of Genioglossus EMG Reflex Using Berkner Transform for Time Latency Measurement in OSA Pathophysiological Studies
}

\author{
Pierre Yves Guméry*, Hervé Roux-Buisson, Sylvain Meignen, François Louis Comyn, Maurice Dematteis, \\ Bernard Wuyam, Jean Louis Pépin, and Patrick Lévy
}

\begin{abstract}
To investigate obstructive sleep apnea syndrome mechanisms, we developed a device to measure the surface electromyogram (EMG) time latency reflex of the genioglossus muscle stimulated by time and amplitude calibrated negative pharyngeal pressure drops. The reflex signals were found to be disturbed by transient signals that generate false alarms. Thus, to reduce false alarm occurrences we designed an adaptive multiscale method. Continuous wavelet transform (CWT) is widely used in biomedical signal event detection processes. The Berkner transform is an approximation of a CWT that is based on a hierarchical scheme similar to discrete wavelet transform. We used the Berkner transform to build a multiscale detector because it offers the possibility of maxima coefficients linkage that leads to good accuracy in reflex onset localization. As a contribution to this novel approach we used a reconstruction formula to develop an adaptive method for scale range determination in our surface EMG reflex detector. Finally, we characterized our detector in terms of accuracy and robustness, first on synthesized signals and second, on signals acquired on apneic patients and healthy subjects. Preliminary results showed a significant difference $(p<0.01)$ between the two populations regarding the genioglossus muscle mean latency time. These physiological findings may partly explain why the upper airway protective reflex occurring when a negative pressure is applied to the upper airway is ineffective in OSA patients, leading to pharyngeal collapse.
\end{abstract}

Index Terms-Continuous wavelet transform (CWT), sleep apnea syndrome, surface electromyography.

\section{INTRODUCTION}

$\mathbf{O}$ BSTRUCTIVE sleep apnea-hypopnea syndrome (OSAS) is a clinical syndrome characterized by recurring episodes of upper airway narrowing and/or closure during sleep. OSAS represents a serious public health problem, since two to four percent of the adult population is affected. Collapse of the upper airway is usually explained by the difference between pharyngeal dilating muscle activity demonstrating inspiratory

Manuscript received August 4, 2003; revised October 31, 2005. Asterisk indicates corresponding author.

*P. Y. Guméry is with the Laboratoire Techniques de l'Imagerie, de la Modélisation et de la Cognition, UMR-CNRS 5525, Joseph Fourier University, 38706 Grenoble, France (e-mail: Pierre-Yves.Gumery@imag.fr).

$\mathrm{H}$. Roux-Buisson is with the Laboratoire Techniques de l'Imagerie, de la Modélisation et de la Cognition, UMR-CNRS 5525, Joseph Fourier University, 38706 Grenoble, France.

S. Meignen is with the Laboratoire de Modélisation et de Calcul, Joseph Fourier University, 38706 Grenoble, France.

F. L. Comyn, M. Dematteis, B. Wuyam, J. L. Pépin, and P. Lévy are with the Laboratoire Hypoxie PathoPhysiologie, Joseph Fourier University and Sleep Laboratory, 38706 Grenoble, France.

Digital Object Identifier 10.1109/TBME.2005.851518 phasic activity, and the collapsing forces, i.e., intraluminal negative pressure, generated by thoracic muscle contraction. The genioglossus is one of the most important pharyngeal dilating muscles phasically active within the breathing cycle [1]. It is well established that applying a negative pressure stimulus on the pharynx both in animals and humans increases genioglossus activity [2]. The short time latency reflex and the reduction of this activity by topical upper airway anaesthesia suggest that genioglossus activation is linked to a reflex mediated by airway mucosal receptors located in the pharynx, namely at the nasopharyngeal level. There is a possible relationship between time latency reflex of the genioglossus muscle activation, oxygen saturation, and apnea syndrome severity [3]. Thus, the measurement of the genioglossus time latency reflex is of interest for understanding OSA pathophysiology. Previous studies have shown that respiratory-related cortical activity may be evoked by airway occlusion [4]-[6] or in response to small, brief negative pressure pulses [7]-[9]. Akay et al. [10] have shown that the evoked response to pressure pulses applied at onset of inspiration is reduced in OSAS subjects. This may reflect reduced sensitivity of the pharyngeal mechanoreceptors or reduced mechanoreceptor stimulation on a more compliant upper airway.

Prior studies [11], [12] have shown that the surface technique may adequately reflect the bioelectric activity of the genioglossus. Horner et al. [11] have measured the normal subject time latency reflex on the rectified and integrated poststimulus surface genioglossus electromyogram (SGEMG) using a threshold of two background EMG activity standard deviation. This approach, referring to "single-threshold techniques," is traditionally proposed for the estimation of on-off timing of muscle activity. These methods are characterized by a large sensitivity to noise. Considering surface EMG onset activity detectors, Merlo et al. [13] underlined recently the usefulness of local signal analysis where global properties are replaced by local ones. They developed a detector based on the continuous wavelet transform (CWT). This solution naturally led to a more accurate estimation of the muscle's on-off timing, that is of interest for time reflex measurement. More generally, multiresolution analysis has been largely applied to other biomedical signals [14]-[17]. Considering the respiratory related evoked responses to pressure pulses, Akay et al. [10] applied wavelet decomposition to improve signal detection and characterization in noisy background. One of the techniques used was a denoising approach based on wavelet maxima. 
In this paper, we designed and evaluated a device aimed at measuring the SEMG time latency reflex of the genioglossus muscle. The genioglossus response was obtained in response to time and amplitude calibrated negative pressure drops applied to the upper airway at the onset of inspiration. We compared the genioglossus time latency for both awake healthy subjects and awake OSAS patients and hypothesized that a significant delay may occur in OSAS patients. Such a delay in genioglossus muscle activation would support the hypothesis that OSAS is characterized by impaired upper airway mechanoreceptor sensitivity or delayed efferent transmission to the upper airway dilating muscles.

We built an adaptive multiscale event detector in SGEMG signal containing artifacts. The events consisted in abrupt changes in signal energy that corresponded to the onset of the genioglossus reflex. To ensure good accuracy in the time onset estimation (localization on the finest considered scale), as well as a robust detection (pertinent scale range choice for artifact rejection), we chose to develop a detector based on the extrema lines (associated to the SGEMG singularities) of the Berkner Transformation (BT) [18] that approximated the CWT when the wavelet kernel was chosen as a derivative of the Gaussian function.

The present paper is organized as follows: in Section II, we present the subjects and the experimental procedures; in Section III, we give a signal description; in Section IV, we present the adaptive detector; in Section V, we analyze the robustness and precision on a phenomenological model of SGEMG reflex. In Section VI, we present results of artifact rejection in experimental signals and we show original preliminary results obtained on healthy subjects and OSAS patients. Finally, in Section VII, we discuss the results and perspectives.

\section{SUBJECTS AND EXPERIMENTAL PROCEDURES}

\section{A. Subjects}

We studied men only, to avoid the influence of the gender factor. A first set of seven healthy men made up the control group. They were free of symptoms and specifically exhibited no excessive daytime sleepiness or snoring, e.g., mean value for the Epworth sleepiness score being $6.7( \pm 2.5)$. The controls had overnight oximetry (Biox, Ohmeda). All subjects had a normal oximetric tracing without any periodic desaturation that could reflect the occurrence of apnea or hypopnea. On average control subjects were $47.4( \pm 6.4)$ years old and their mean body mass index (BMI) was $24.2( \pm 2.5) \mathrm{kg} / \mathrm{m}^{2}$. No subject exhibited neurological, cardiovascular, or pulmonary symptoms. A second set of seven subjects included OSAS patients who had a complete diagnostic polysomnography at the Grenoble University Sleep Laboratory. No patient was under treatment. Their mean Epworth score was $9( \pm 2.8)$. On average, they were 53 $( \pm 13.4)$ years old and their mean BMI was $30( \pm 5.3) \mathrm{kg} / \mathrm{m}^{2}$. Overall they had severe OSAS [mean AHI:53.7 ( \pm 26.3$)]$. The mean polysomnographic data of OSAS patients are provided in Table I.

\section{B. Experimental Setup}

The experimental device allowed a calibrated negative pressure pulse to be applied at the onset of inspiration, and to measure the SGEMG signal and the mask pressure simultaneously.
TABLE I

MEAN POLYSOMNOGRAPHIC DATA OF OSAS PATIENTS. AHI, APNEA-HYPOPNEA INDEX; AI, APNEA INDEX; OAI, OBSTRUCTIVE APNEA InDEX; MAI, MiXED APNEA INDEX; HI, HyPOPNEA INDEX; CAI, CEnTRAL APNEA INDEX

\begin{tabular}{|c|c|c|c|c|c|c|c|c|c|}
\hline \multirow[b]{2}{*}{$\begin{array}{c}O S A S \\
\text { PATIENTS } \\
\end{array}$} & \multirow{2}{*}{$\begin{array}{l}\text { Sleep } \\
\text { Sa02 } \\
\text { mean }\end{array}$} & \multirow{2}{*}{$\begin{array}{l}\text { Sleep } \\
\text { Sa02 } \\
\text { min } \\
\end{array}$} & \multirow{2}{*}{$\begin{array}{c}\% \\
\text { Sa02 } \\
<90 \% \\
\end{array}$} & \multirow[b]{2}{*}{ AHI } & \multirow[b]{2}{*}{$\mathbf{A I}$} & \multirow[b]{2}{*}{ OAI } & \multirow[b]{2}{*}{ MAI } & \multirow[b]{2}{*}{ HI } & \multirow[b]{2}{*}{ CAI } \\
\hline & & & & & & & & & \\
\hline Mean & 91.6 & 63.0 & 29.2 & 53.7 & 18.5 & 9.4 & 13.3 & 19.0 & 0.8 \\
\hline SD & 2.6 & 25.9 & 26.1 & 26.3 & 14.9 & 13.1 & 19.7 & 16.9 & 1.0 \\
\hline
\end{tabular}

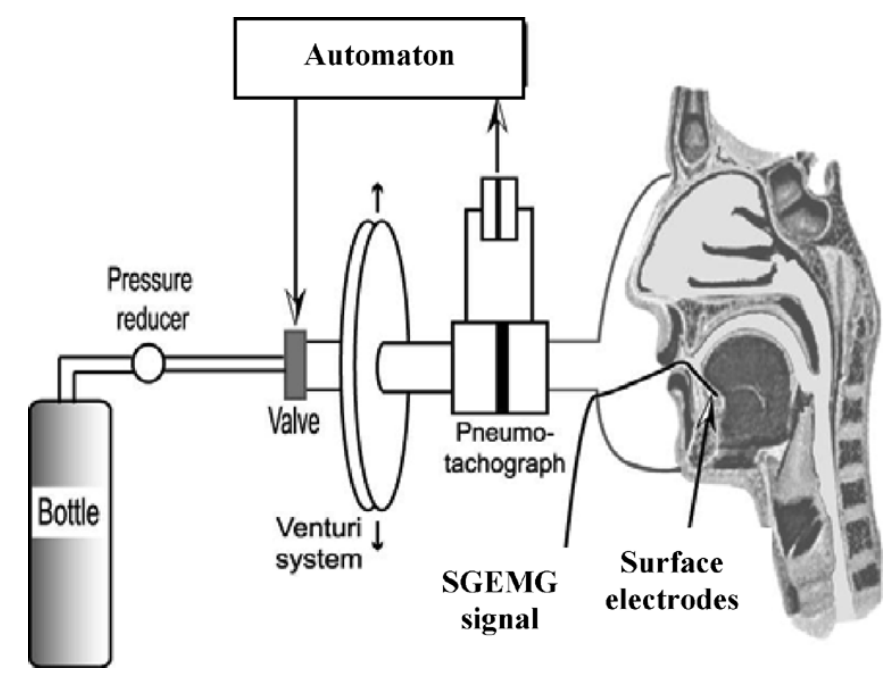

Fig. 1. The experimental device.

The SGEMG Sensor: The gathering of the SGEMG activity was carried out with one pair of electrodes on each side of the sagittal plane near the lingual frenulum. For each subject, these electrodes were fixed on sheet of polymer, warm-pressed on a plaster copy of the lower denture and the floor of the mouth. Thus, the electrode supports were well adapted to the anatomical characteristics of each subject. The positioning of electrodes on the support was chosen to ensure the best contact. The SGEMG was amplified 10000 times using an INA 101 Burr-Brown instrumentation amplifier, filtered by an analogue second-order bandpass filter $(20-400 \mathrm{~Hz})$. A 16-bit Biopac MP100 system was used for signal acquisition. The sampling rate was fixed at $1000 \mathrm{~Hz}$. The electronic noise level was estimated in three cases: 1) human saliva; 2) sodium chloride solutions of $50 \mathrm{meq} / \mathrm{L}$; and 3) sodium chloride solutions of $250 \mathrm{meq} / \mathrm{L}$ [12]. For these three cases the noise level was found, respectively, to be equal to 5 $\mu \mathrm{Vrms}, 2 \mu \mathrm{Vrms}$ and $2 \mu \mathrm{Vrms}$. For all subjects, the amplitude of the SGEMG tonic background activity (i.e., the prestimulus SGEMG part) was at least four times that of the electronic noise.

The Negative Pressure Pulse Generator and Mask Pressure Measurement (Fig. 1): A compressed air bottle supplied a Venturi device (Aeromech Devices, Almonte, ON, Canada) via a pressure reducer followed by a coil valve driven by an automaton (Direct Physiologic Recording System). Pressure between 1 and 2 bars was applied to the Venturi which yielded negative mask pressures between -6 and $-15 \mathrm{cmH}_{2} \mathrm{O}$. This mask pressure was measured with an EXAR SM56-52 sensor. The coil valve was triggered at the onset of inspiration. The 

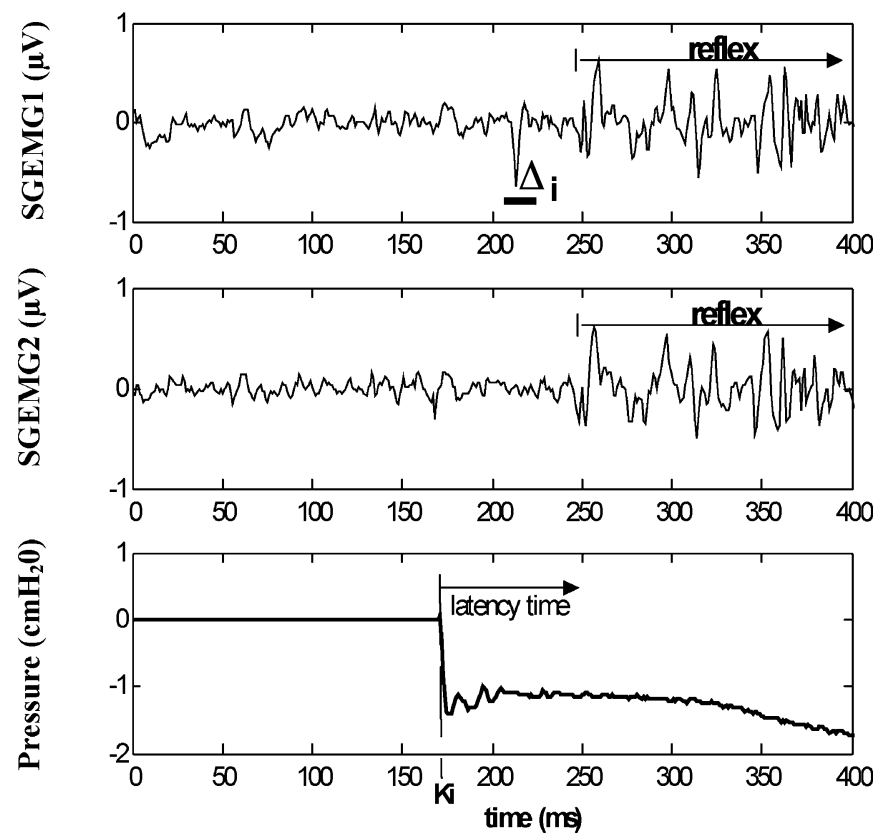

Fig. 2. Bichannel SGEMG record. (a)-(b) SGEMG reflex response. (c) Pressure stimulus.

pressure signal was amplified 170 times and low-pass filtered with a cutoff frequency of $340 \mathrm{~Hz}$. Fig. 2 shows the pressure stimulus and the SGEMG reflex. On this recording we used a bichannel intraoral device. The signals were very similar on the two channels as electrodes were placed very close to each other. Fig. 2(a) shows that electrode instabilities could generate transient artifacts $\left(\right.$ see $\left.\Delta_{i}\right)$.

\section{Stimuli Protocol}

The stimulation set included 30 stimuli applied in the supine position and 30 stimuli applied in the seated position. For each position we applied 10 stimuli at three different negative pressure levels: 6,10 , and $15 \mathrm{cmH}_{2} \mathrm{O}$. The pressure pulse duration was fixed at $500 \mathrm{~ms}$.

\section{Statistical Analysis}

Considering the small number of subjects, the statistical analysis was completed using the nonparametric U test of MannWhitney. Data were expressed as means \pm SD. Statistical significance was accepted when $\mathrm{p}<0.05$.

\section{SIGNAL DESCRIPTION}

Surface EMG is a summation of motor unit action potential (MUAP) trains. Using a prototype function $\mathrm{f}(\mathrm{t})$ that describes MUAP shape, a signal model can be described as follows:

$$
\operatorname{MUAPT}_{\mathrm{j}}(\mathrm{t})=\sum_{\mathrm{i}} \mathrm{k}_{\mathrm{j}} . \mathrm{f}\left(\frac{\mathrm{t}-\tau_{\mathrm{ij}}}{\alpha_{\mathrm{j}}}\right)
$$

where $\mathrm{j}$ indicates a specific motor unit, $\mathrm{k}_{\mathrm{j}}$ is an amplitude factor, and $\tau_{\mathrm{ij}}$ is the occurrence times of MUAPs of the motor unit. $\alpha_{\mathrm{j}}$ represents a scaling factor [13]. According to this, one can see that different MUAP have the same shape but different widths and amplitudes. The EMG signal can be expressed as

$$
\operatorname{EMG}(t)=\sum_{j} \operatorname{MUAPT}_{j}(t)
$$

At rest in the prestimulus period, SGEMG signal picked up by the electrodes was made up of the background EMG activity described by (2) and called $b(t)$; at the reflex occurrence the signal became $\mathrm{r}(\mathrm{t})$ also described by (2) but with a greater amplitude. The frequency band of $b(t)$ and $r(t)$ was found to be the same. The SGEMG was affected by random transient artifacts which occurred on average twice every second. Their duration was between 2 and $20 \mathrm{~ms}$. Their occurrence probability was time independent, thus they conformed to Poisson's process and the overlapping probability was lower than $4.10^{-4}$. Their amplitude might have been greater than that of the reflex signal. We separated these transients into two classes: the first $\Delta_{\mathrm{i} 1}(\mathrm{t})$ with a very similar frequency content to that of the SGEMG and the second $\Delta_{\mathrm{i} 2}(\mathrm{t})$ with a lower frequency content than that of the SGEMG. Finally the whole signal can be written as

$$
\mathrm{s}(\mathrm{t})=\mathrm{b}(\mathrm{t})+\mathrm{r}(\mathrm{t})+\sum_{i} \Delta_{i 1}(t)+\sum_{i} \Delta_{i 2}(t)
$$

\section{Detection Method}

In the SGEMG signal, singularities correspond to the gradient change associated to the edges of the MUAP that make up the signal. For singularity detection and localization, it is sufficient to consider only the first derivative of the Gaussian Kernel. The scale range choice is an important problem in multiscale analysis and it is currently performed on a priori assumptions or empirical criteria. In this section we propose a numerical criterion based on a reconstruction formula [19], [20] to elaborate an adaptive scale range determination.

\section{A. The Berkner Transform}

The BT is an approximation of the Gaussian derivative wavelet transform based on a hierarchical scheme similar to the popular fast discrete wavelet transform [18]-[20]. It is easy to compute and a simple relation [19] enabled us to follow the extrema lines in the time scale plane. More precisely, BT was achieved through the discrete convolution of a signal $s$ with the following approximation of the first derivative of a Gaussian [18]

$$
\rho_{N}(k)=2^{-N}\left(1-\frac{k}{N-k+1}\right) C_{k}^{N}
$$

with

$$
C_{k}^{N}=\frac{N !}{k !(N-k) !}
$$

where $k$ is the sampled time and $N$ the scale of the wavelet. From (4) it is easy to demonstrate that

$$
\rho_{N}(k)=\frac{1}{2}\left[\rho_{N-1}(k)+\rho_{N-1}(k-1)\right] .
$$


Owing to this, it is possible to follow an extremum from scale $\mathrm{N}$ to scale $\mathrm{N}+1$. In [19] we proposed a modified Berkner expansion

$$
\bar{c}_{N}(k)=\sum_{j=0}^{\infty} \bar{\rho}_{N}(k) s(k+j)
$$

with

$$
\bar{\rho}_{N}(k)=\bar{\rho}_{N}\left(k+\left\lfloor\frac{N+1}{2}\right\rfloor\right)
$$

so the recurrence relation (5) was also modified and led to

$$
\begin{aligned}
& \bar{c}_{N}(k)=\frac{1}{2}\left(\bar{c}_{N-1}(k)+\bar{c}_{N-1}(k-1)\right), \text { if } \mathrm{N}+1 \text { was even } \\
& \bar{c}_{N}(k)=\frac{1}{2}\left(\bar{c}_{N-1}(k)+\bar{c}_{N-1}(k+1)\right), \text { if } \mathrm{N}+1 \text { was odd. }(7)
\end{aligned}
$$

\section{B. Reconstruction Process}

From (4) and (6) one can show [19] that

$$
\mathrm{s}(\mathrm{k})=\frac{1}{2} \sum_{l=0}^{\infty} \bar{c}_{l}\left(k+\left\lfloor\frac{l+1}{2}\right\rfloor\right)
$$

which is the exact form of the reconstruction formula. An approximated value will be obtained by summing the first $\mathrm{N}+1$ values of (8) that is

$$
\mathrm{S}_{\mathrm{a}}(\mathrm{k})=\frac{1}{2} \sum_{l=0}^{\mathrm{N}} \bar{c}_{l}\left(k+\left\lfloor\frac{l+1}{2}\right\rfloor\right) .
$$

Another approximation may be obtained using only the local extrema coefficients. Let us define

$$
\overline{\mathrm{C}}_{l}\left(k+\left\lfloor\frac{l+1}{2}\right\rfloor\right)=\bar{c}_{l}\left(k+\left\lfloor\frac{l+1}{2}\right\rfloor\right)
$$

for $\bar{c}$ extremum and $\overline{\mathrm{C}}_{l}=0$ elsewhere.

So the approximate reconstructed signal is

$$
\mathrm{S}_{\mathrm{a}}(\mathrm{k})=\sum_{l=0}^{\mathrm{N}} \overline{\mathrm{C}}_{l}\left(k+\left\lfloor\frac{l+1}{2}\right\rfloor\right) .
$$

We define the reconstruction error as

$$
\ell_{2 m}=\frac{\sum(\mathrm{s}(\mathrm{k})-\mathrm{Sa}(\mathrm{k}))^{2}}{\sum(s(k))^{2}}
$$

and

$$
\ell_{2}=\frac{\sum\left(\mathrm{s}(\mathrm{k})-\mathrm{s}_{\mathrm{a}}(\mathrm{k})\right)^{2}}{\sum(s(k))^{2}}
$$

where the sums are extended to the definition domain of $\mathrm{s}(\mathrm{k})$. One can see on Fig. 3 that $\ell_{2}$ vanished when $\mathrm{N}$ increased and $\ell_{2 m}$ exhibited a minimum for $\mathrm{N}=\mathrm{N}_{0}$ and then remained constant. This behavior can be explained by the small size of the extrema coefficients when the values of $\mathrm{N}$ higher than $\mathrm{N}_{0}$. In
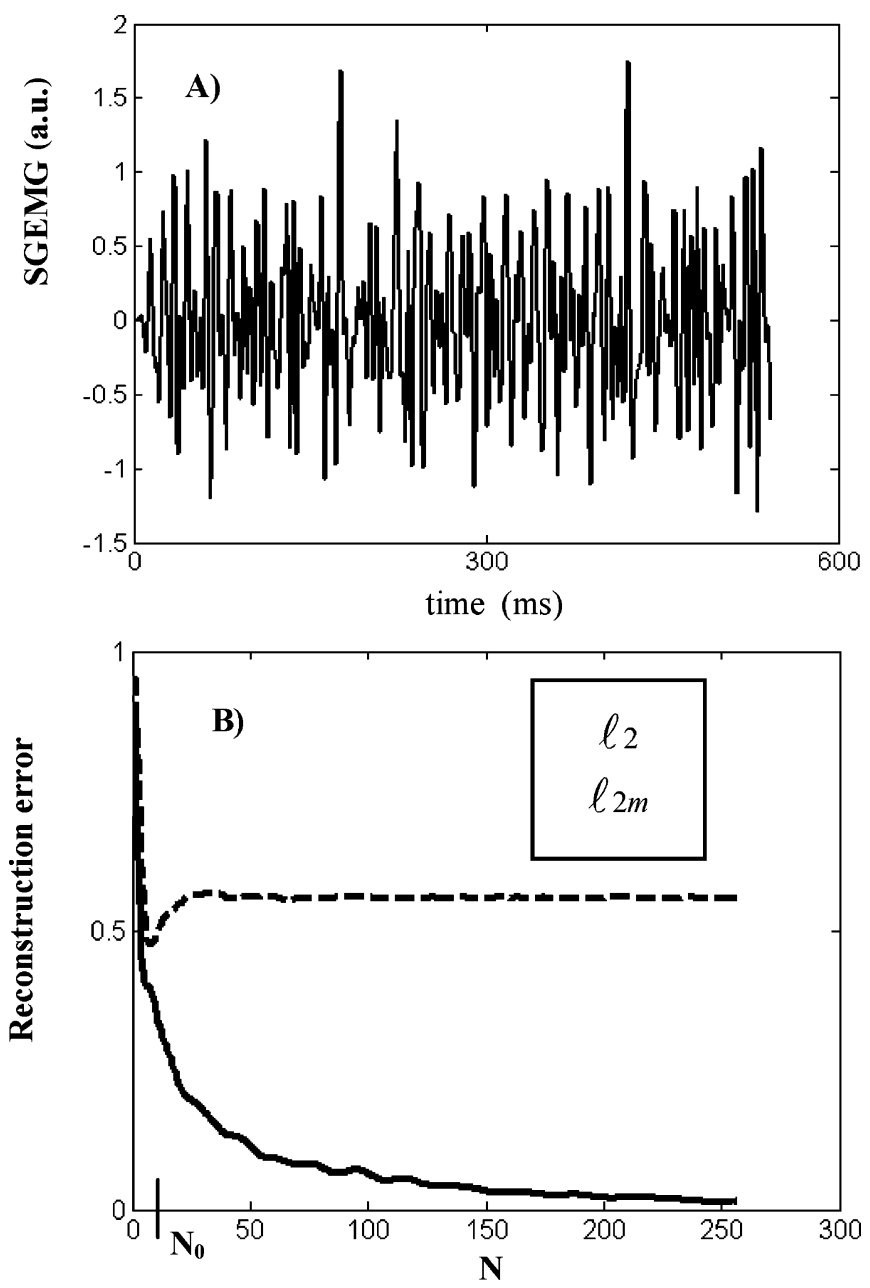

Fig. 3. (a) SGEMG background signal (a.u.: arbitrary unit). (b) Reconstruction errors versus $\mathrm{N}$.

the following, we describe the use of this property to build an adaptive scale determination process.

\section{Detection}

The signal presented three possible states corresponding to the following:

1) the background activity: hypothesis $\mathrm{H}_{1}$;

2) the background disturbed by artifacts: hypothesis $\mathrm{H}_{2}$;

3) the reflex activity: hypothesis $\mathrm{H}_{3}$.

We wanted to characterize these three states statistically in order to distinguish between them. First we determined the value $\mathrm{N}_{0}$ associated with $b(t)$. Consequently as shown in Section IV, this method enabled us to eliminate $\Delta_{i 2}$ transients. The $\mathrm{b}(\mathrm{t})$ signal was considered as Gaussian and stationary during $500 \mathrm{~ms}$ before the stimulus and during the latency time. The statistical characteristics of the signal $b(t)$ as well as the scale $\mathrm{N}_{0}$ were determined over a $75-\mathrm{ms}$ window extracted from the above $500 \mathrm{~ms}$. In the prestimulus period, only $\mathrm{H}_{1}$ or $\mathrm{H}_{2}$ were possible. The probability of $\mathrm{H}_{1}$ was $P_{1}=\exp \left(T_{p} / \alpha\right)$ where $\mathrm{T}_{\mathrm{p}}$ was the evaluation window and $\alpha$ the mean artifact occurrence; with $\alpha=.5 \mathrm{~s}$ and $\mathrm{T}_{\mathrm{p}}=75 \mathrm{~ms}$ we found $\mathrm{P}_{1}=.86$. Thus the prestimulus period allowed us to characterize the background in most cases. 
However, in $14 \%$ of cases the presence of artifacts biased the characterization. By applying Kolmogorov's test, we were able to detect this presence and through a simple threshold we eliminated the artifact by truncation and consequently suppressed the bias. In the former case, the probability of encountering only one artifact was 0.13 . Finally, in $99 \%$ of the cases, the background was characterized without bias. In the remaining $1 \%$, the signal was not processed and another evaluation window was chosen. Once the stimulus was applied we had to detect when we left the hypothesis $\mathrm{H}_{1}$. We did that by using an energy threshold on extrema lines. We used only the extrema coefficients because they reflect the singularities generated by MUAP trains, and they are thus relevant to the events to be detected. In fact we used all the extrema lines which reflect the same event at different scales. A simple energy criterion could not separate $\mathrm{H}_{2}$ from $\mathrm{H}_{3}$. Fortunately, transient artifacts i.e., $\mathrm{H}_{2}$ generated lower density of maxima lines than reflexes, i.e., $\mathrm{H}_{3}$. Thus, we introduced a threshold on the number of maxima lines in a given window $w$ to separate $\mathrm{H}_{2}$ from $\mathrm{H}_{3}$. We will now set out the principles just stated.

Step 1) Rejection of hypothesis $H_{1}$ :

Let $\mathrm{L}$ be the set of the extrema lines in the prestimulus period and $\ell$ be one of these lines. The variable

$$
\mathrm{D}_{\ell_{p}}=\sum_{(k, j) \in \ell, j<p} \overline{\mathrm{C}}_{j}^{2}(k)
$$

was $\sigma^{2} \chi_{2}$ distributed. Degrees of freedom $\nu_{p}$ and variance $\sigma_{p}^{2}$ were estimated by the method of moments

$$
\sigma_{p}^{2}=\frac{\operatorname{var}\left(\mathrm{D}_{\ell_{p}}\right)}{2\left\langle\mathrm{D}_{\ell_{p}}\right\rangle} \quad \text { and } \quad \nu_{p}=\left\lceil\frac{2\left\langle\mathrm{D}_{\ell_{p}}\right\rangle}{\operatorname{var}\left(\mathrm{D}_{\ell_{p}}\right)}\right\rceil
$$

where $\left\lceil 2\left\langle\mathrm{D}_{\ell_{p}}\right\rangle / \operatorname{var}\left(\mathrm{D}_{\ell_{p}}\right)\right\rceil$ denotes the nearest integer of the term $2\left\langle\mathrm{D}_{\ell_{p}}\right\rangle / \operatorname{var}\left(\mathrm{D}_{\ell_{p}}\right)$ and $\left\langle\mathrm{D}_{\ell_{p}}\right\rangle$ the mean value of $\mathrm{D}_{\ell_{p}}$. If $\mathrm{D}_{\ell_{p}}$ was greater than a given threshold $\lambda_{p}$ this value did not follow the estimated law with probability $\mathrm{P}_{\mathrm{r}}$. We considered that a line $\ell$ with length $\mathrm{p}_{\ell}$ verified $\mathrm{H}_{0}$ if $\mathrm{D}_{\ell_{p}}$ verified the chi-squared distribution for more than half the value of $\mathrm{p}$. This can be expressed

$$
\frac{\operatorname{card}\left(\left\{p \leq \min \left(N_{0}+1, p_{\ell}\right), \mathrm{D}_{\ell_{p}} \geq \lambda_{p}\right\}\right)}{\min \left(N_{0}+1, p_{\ell}\right)} \leq 0.5
$$

where card means the cardinal of the set defined in the parentheses. We detail now the decision between $\mathrm{H}_{2}$ and $\mathrm{H}_{3}$.

Step 2) Rejection of hypothesis $\mathrm{H}_{2}$ :

Experiments showed that artifacts, unlike reflexes, generated fewer than five extrema lines in twenty milliseconds. Thus, when a line $\ell$ appeared at the discrete time $\mathrm{k}_{l}$, which did not verify $\mathrm{H}_{0}$, we counted the number of lines that did not verify $\mathrm{H}_{0}$ in the detection window $w=20 \mathrm{~ms}$ (i.e., 20 samples). If the number of such lines was greater than $5, \mathrm{H}_{2}$ was rejected. This can be written as follows:

$\operatorname{card}\left(\overline{\ell_{H 0}^{k}}, k \in\left\{k_{l}, \ldots, k_{l}+w\right\}\right) \geq 5$

where $\overline{\ell_{H 0}^{k}}$ represents an extrema line that does not verify $\mathrm{H}_{0}$ whose lowest scale is located at time $\mathrm{k}_{l}$. This time corresponded to the estimated reflex onset time.

\section{Detector Characterization on Synthesized Signals}

\section{A. Signal Synthesis}

We had two possible choices to simulate SGEMG. The first one was a phenomenological model that led to a stochastic signal. The second one was based on a physiological and physical model taking into account the MUAP wave shape. The latter method needed strong a priori assumptions on electrode placement which were not verified by our customized probe. Moreover, as we wanted to detect only singularities, a phenomenological approach was appropriate. Synthesized signals were made of autoregressive (AR) models from experimental signals in the background state without transient artifacts. These signals were gathered in the set of seven apneic patients and in the set of seven healthy subjects. Finally, we built 20 different AR models in the whole set of background signals. To evaluate the event localization capabilities of our detector, we chose to model reflex onsets as instantaneous abrupt amplitude changes. The SGEMG simulation due to the ith pressure stimulus at time $\mathrm{k}_{\mathrm{i}}$ was carried out as follows: during a time $\mathrm{T}_{1}$ before the stimulus, up to a time $\tau$ after the stimulus, the signal was considered as background activity; then the reflex activity was simulated, multiplying the background activity by an adjustable coefficient $\Delta_{\text {amp }}$ during a time $\mathrm{T}_{2}$. Hence, this could be expressed in a discrete way as

$$
\begin{aligned}
& k \in\left[k_{i}-T_{1}, k_{i}\right] \text { Then } f(k)=b(k) \\
& k \in\left[k_{i}, k_{i}+\tau\right] \text { Then } f(k)=b(k) \\
& k \in\left[k_{i}+\tau, k_{i}+T_{2}\right] \text { Then } f(k)=\Delta_{\text {amp }} . b(k)=r(k) .
\end{aligned}
$$

During $\mathrm{T}_{1}=75 \mathrm{~ms}$ we estimated the background features. The latency time $\tau$ was chosen equal to $75 \mathrm{~ms}$, which was close to the mean value of experimental latency time measured on control set (see Section VI).

\section{B. Detector Performance Analysis on Signals Without Artifacts}

We tested the influence of the parameters $\mathrm{P}_{\mathrm{r}}$ and $\Delta_{\mathrm{amp}}$ on the following:

- the probability of non detection (PND);

- the probability of detection (PD);

- the probability of false alarm (PFA).

Let us define $\tau_{\text {esti }}$ as the measured value of $\tau$ for the ith synthesized reflex response. False alarm was defined by $\tau_{\text {esti }} \in$ $[0, \tau-w]$, detection by $\tau_{\text {esti }} \in[\tau-w, \tau+w]$ and nondetection in the other cases or when the detection was not performed. Within these conditions PND + PFA + PD $=1$. Table II shows the results obtained for 200 simulations (i.e., 10 per AR model) on PND and PD versus $P_{r}$ and $\Delta_{a m p}$. We can infer from these values that no false alarm appeared. This result can be explained by the use of window $w$ that ensured a low FA rate. We 
TABLE II

(a) Probability of Non Detection Versus $\Delta_{\text {amp }}$ AND $P_{r}$. (b) Probability of Detection Versus $\Delta_{\text {amp }}$ AND $P_{r}$

\begin{tabular}{c|c|c|c|c|c}
\hline PND & $\Delta a m p$ & $\Delta a m p$ & $\Delta a m p$ & $\Delta a m p$ & $\Delta a m p$ \\
$=1.2$ & $=1.5$ & $=1.8$ & $=2.1$ & $=2.4$ \\
\hline$P_{r}=0.95$ & 0.9000 & 0 & 0 & 0 & 0 \\
\hline$P_{r}=0.99$ & 0.9100 & 0 & 0 & 0 & 0 \\
\hline$P_{r}=0.999$ & 0.9150 & 0 & 0 & 0 & 0 \\
\hline$P_{r}=0.9999$ & 0.8550 & 0 & 0 & 0 & 0 \\
\hline
\end{tabular}

(a)

\begin{tabular}{c|c|c|c|c|c}
\hline $\mathrm{PD}$ & $\Delta a m p$ & $\Delta a m p$ & $\Delta a m p$ & $\Delta a m p$ & $\Delta a m p$ \\
$=1.2$ & $=1.5$ & $=1.8$ & $=2.1$ & $=2.4$ \\
\hline$P_{r}=0.95$ & 0.100 & 1.0000 & 1.0000 & 1.0000 & 1.0000 \\
\hline$P_{r}=0.99$ & 0.0900 & 1.0000 & 1.0000 & 1.0000 & 1.0000 \\
\hline$P_{r}=0.999$ & 0.0850 & 1.0000 & 1.0000 & 1.0000 & 1.0000 \\
\hline$P_{r}=0.9999$ & 0.1450 & 1.0000 & 1.0000 & 1.0000 & 1.0000 \\
\hline
\end{tabular}

(b)

chose a low FA rate because FA affected the latency time estimation. The ND did not modify these estimations but required an additional stimulation. Table II shows that from the value $\Delta_{\mathrm{amp}}=1.5$, the detection was certain for the considered values of $\mathrm{P}_{\mathrm{r}}$. After the evaluation of the robustness, we characterized the accuracy of the time latency measurement. For $\mathrm{P}_{\mathrm{r}}=.99$ and $\Delta_{\mathrm{amp}}=1.5$, Table III shows that the standard deviation reached a few milliseconds and the gap between the expected and the measured value was approximately $1 \mathrm{~ms}$.

\section{RESULTS ON EXPERIMENTAL SIGNALS}

\section{A. Artifact Considerations}

We show on signals strongly disturbed by artifacts (Fig. 4) that step 1 of our detector allowed the rejection of $\Delta_{i 2}$ transients.

On the other hand, the remaining $\Delta_{i 1}$ artifacts could modify the time latency measurements when they occurred $20 \mathrm{~ms}(w$ duration) or less before the SGEMG reflex response. In this case, the latency time measured, decreased. The wider the window was, the larger the error was. Taking into account Poisson's law, the occurrence probability of at least one artifact in a 20-ms window $w$ was 0.04 . The maximum of induced error was $20 \mathrm{~ms}$, which was quite large compared to the results of Table III. In fact, as the error was uniformly distributed on $w$, its mean value was $10 \mathrm{~ms}$. As the statistical weight of the erroneous values was weak, i.e., $4 \%$, the induced mean error $(\mathrm{n}=10)$ remained lower than $2 \mathrm{~ms}$.

\section{B. Preliminary Results for OSAS Patients and Healthy Subjects}

Given that the negative pressure drop was triggered at the onset of the inspiration, we had to eliminate records that exhibited an important phasic inspiratory activity and that could not
TABLE III

(a) MEAn ERror on Time Latency MeAsurements Versus $\Delta_{\text {amp }}$ AND $P_{r}$. (b) StAndard DEVIATION VeRsus $\Delta_{\text {amp }}$ AND $P_{r}$

\begin{tabular}{c|c|c|c|c|c}
\hline mean & $\Delta a m p$ & $\Delta a m p$ & $\Delta a m p$ & $\Delta a m p$ & $\Delta a m p$ \\
& $=1.2$ & $=1.5$ & $=1.8$ & $=2.1$ & $=2.4$ \\
\hline$P_{r}=0.95$ & 23.0000 & 1.0300 & 1.3050 & 1.4300 & 1.4300 \\
\hline$P_{r}=0.99$ & 22.5789 & 0.7025 & 1.1200 & 1.2250 & 1.2250 \\
\hline$P_{r}=0.999$ & 21.7091 & 0.4800 & 1.0200 & 1.1067 & 1.1067 \\
\hline$P_{r}=0.9999$ & 22.1071 & 0.2700 & 0.9762 & 1.0438 & 1.0438 \\
\hline
\end{tabular}

(a)

\begin{tabular}{c|c|c|c|c|c}
\hline std & $\Delta a m p$ & $\Delta a m p$ & $\Delta a m p$ & $\Delta a m p$ & $\Delta a m p$ \\
& $=1.2$ & $=1.5$ & $=1.8$ & $=2.1$ & $=2.4$ \\
\hline$P_{r}=0.95$ & 15.5969 & 3.4099 & 1.7078 & 1.7666 & 1.7666 \\
\hline$P_{r}=0.99$ & 14.0147 & 2.9250 & 1.3785 & 1.4455 & 1.4455 \\
\hline$P_{r}=0.999$ & 13.4669 & 2.6091 & 1.1656 & 1.2211 & 1.2211 \\
\hline$P_{r}=0.9999$ & 13.3001 & 2.3631 & 1.0330 & 1.0820 & 1.0820 \\
\hline
\end{tabular}

(b)

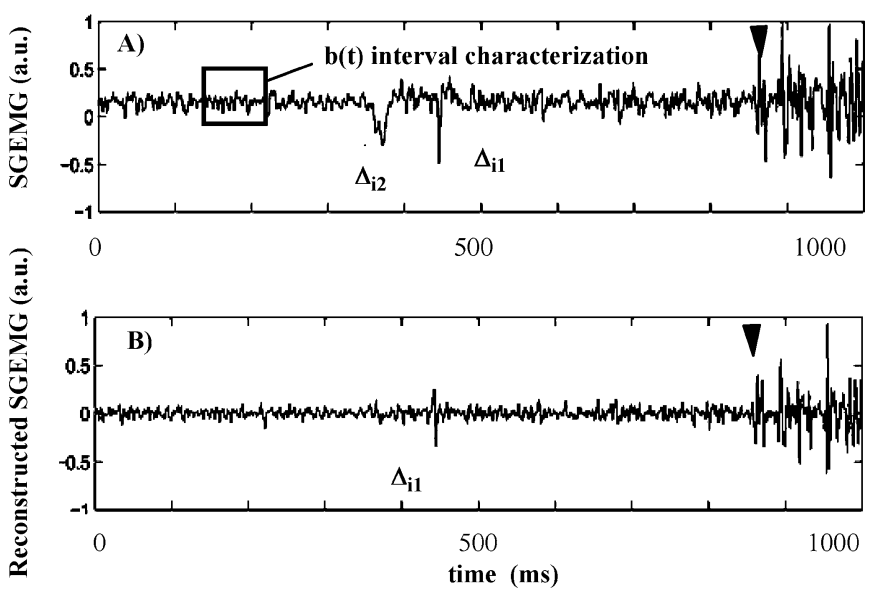

Fig. 4. (a) Original SGEMG signal containing artifacts with reflex event. (b) Reconstructed signal calculated with BT maxima from scales up to $\mathrm{N}_{0}$; one can see the $\Delta_{i 2}$ transient suppression. The arrows indicate onset of the reflex response.

verify the detector hypotheses. We did not take into account latency time measurements that were too close to the latency time of voluntary contractions. This time was evaluated, for each subject, at the beginning of the experiment. Finally, in our results, we only took into account subjects in whom at least 10 reflex detections were obtained on the 60 stimulations. The results obtained for $\mathrm{P}_{\mathrm{r}}=.99$ are shown on Fig. 5. It can be seen that we selected only five healthy subjects and five OSAS patients. The measurements on healthy subjects $\mathrm{H} 6, \mathrm{H} 7$ and OSAS patients P6, P7 were not available (NA). Subjects H6 and P6 exhibited 


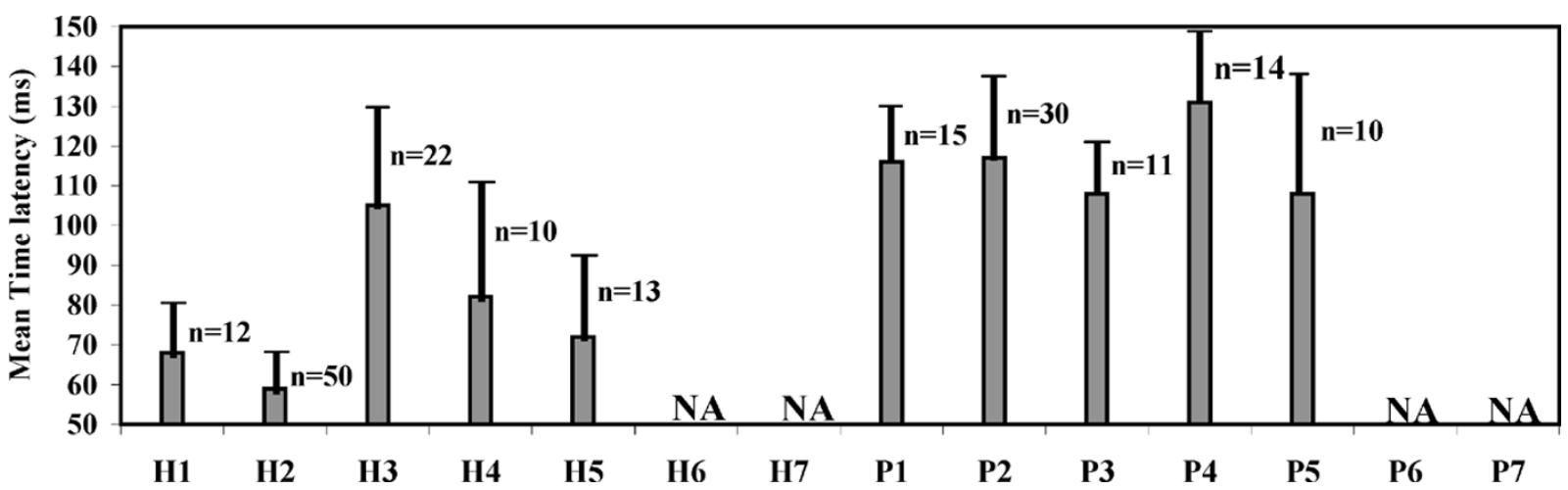

Fig. 5. Mean reflex time latency and standard deviation for each healthy subject $(\mathrm{H})$ and each apneic patient $(\mathrm{P})$. $\mathrm{n}$ indicates the detection reflex number taken into account.

a prohibitive phasic activity and subjects $\mathrm{H} 7$ and $\mathrm{P} 7$ did not exhibit enough detectable reflex responses. On the detection set, the mean time latency reflex for healthy subjects was found to be equal to $77 \pm 18 \mathrm{~ms}$. This was lower than the OSAS patient value which was found to be $116 \pm 10 \mathrm{~ms}$. The difference between the mean latency time of the two populations was found to be significant with the U-test $(p=0.009)$.

\section{DISCUSSION}

In this paper, the singularity detection principle is close to that used recently by Arikidis et al. [21]. They developed an interscale wavelet maximum method to assist in the diagnosis of neuromuscular disease. The intramuscular EMG signals were decomposed using a redundant dyadic wavelet transform. The wavelet chosen was the first derivative of the cubic B-spline that was, like the first derivative of the Gaussian function, well adapted to signal gradient change detection. The method was based on the fact that each rising edge of MUAP is represented on each decomposition scale by a wavelet coefficient maximum.

From a broader point of view, regarding the detection of EMG activity in a noisy background, it is of interest to underline that BT may be used as an EMG matched wavelet transform. Recently, Merlo et al. [13] pointed out the advantages of detectors based on the identification of the MUAP from surface EMG, especially in bad signal-to-noise conditions. In fact, they used a matched CWT, based on a mother wavelet chosen according to the MUAP shape. The method used the fact that in the context of single differential EMG recording, the first-order Hermite-Rodriguez function $\mathrm{HR}_{1}(\mathrm{t})$ is well suited to describe the basic MUAP shape [22] and thus to construct the mother wavelet. The range of the scale parameter is chosen in order to obtain wavelet support within the physiological MUAP durations. As $\mathrm{HR}_{1}(\mathrm{t})$ is proportional to the first derivative of the Gaussian function, we can use the BT as an approximation of the EMG matched CWT. The matched CWT technique is useful to detect MUAP trains in additive noise, but is based on strong a priori electrode placement assumptions and its performances decrease when MUAP shape changes significantly (proximity to innervation zone or tendon region). In the case of SGEMG, such assumptions do not seem reasonable.

The study on synthesized signals has shown that our detector exhibited high robustness and accuracy. However it can be seen on Fig. 5 that the standard deviation of the latency estimated for each subject was greater than that obtained in the simulations.
The major reason that can be suggested to explain this difference is the variability occurring in each subject. Wheatley et al. [23], who used a rectified and electronically integrated (50 ms) intra-muscalar genioglossus EMG, reported that in normal subjects, reflex activation by inspiratory negative pressure pulses was delayed from $53.8 \pm 11.5$ [SEM] $\mathrm{ms}$ in wakefulness $(\mathrm{n}=$ $6)$ to $132.7 \pm 24.5 \mathrm{~ms}(\mathrm{n}=3)$ during NREM sleep. The mean and SEM wakefulness values are in accordance with the results obtained in our control group.

The present preliminary results on healthy subjects and OSAS patients demonstrate that the experimental protocol and the signal processing method lead to an appropriate time resolution. This is accurate enough to evidence the genioglossus muscle activation delay in OSAS patients. From a pathophysiological point of view, our results support a relationship between OSA syndrome and the reflex latency time of the genioglossus. These results suggest an increase in latency time in awake apneic patients. Further studies on larger populations are required in order to confirm these results; however they support the hypothesis that patients suffering from OSAS may exhibit abnormal upper airway load-compensating reflexes during wakefulness.

However, the large number of rejected stimulations due to a high level of phasic activity is a serious limitation of the method. Thus, other within-breath timing for stimulus application, as well as the influence of body position and the range of applied pressure have to be analyzed in further studies.

\section{REFERENCES}

[1] J. E. Remmers, W. J. Degroot, E. K. Sauerland, and A. M. Anch, "Pathogenesis of upper airway occlusion during sleep," J. Appl. Physiol., vol. 44, pp. 931-938, 1978.

[2] R. L. Horner, J. A. Innes, H. B. Holden, and A. Guz, "Afferent pathway(s) for pharyngeal dilator reflex to negative airway pressure in man: a study using upper airway anesthesia," J. Physiol. (London), vol. 436, pp. 31-34, 1991 .

[3] M. Dematteis, J. L. Pépin, M. Jeanmart, C. Deschaux, A. Labarre-Vila, and P. Lévy, "Charcot-Marie-Tooth disease and sleep apnoea syndrome: a family study," Lancet, vol. 357, pp. 267-272, Jan. 2001.

[4] P. W. Davenport, W. A. Freedman, F. J. Thompson, and O. Franzen, "Respiratory-related cortical potentials evoked by inspiratory occlusion in humans," J. Appl. Physiol., vol. 60, pp. 1843-1848, 1986.

[5] A. L. Garden, "Myogenic and scalp signals evoked by midinspiratory airway occlusion," J. Appl. Physiol., vol. 89, pp. 422-429, 2000.

[6] W. Revelette and P. Davenport, "Effects of timing of inspiratory occlusion on cerebral evoked potentials in humans," J. Appl. Physiol., vol. 68, pp. 282-288, 1990.

[7] J. A. Daubenspeck, L. M. Lim, and M. Akay, "Global field power helps to separate respiratory-related evoked potentials from electromyographic contamination," J. Appl. Physiol., vol. 88, pp. 282-290, 2000. 
[8] L. M. Lim, M. Akay, and J. A. Daubenspeck, "Identifying respiratoryrelated evoked potentials," IEEE Eng. Med. Biol. Mag., vol. 14, no. 2, pp. 174-178, Mar.-Apr. 1995.

[9] R. J. Strobel and J. A. Daubenspeck, "Early and late respiratoryrelated potentials evoked by pressure pulse stimuli applied at the mouth in humans," J. Appl. Physiol., vol. 74, pp. 1484-1491, 1993.

[10] M. Akay, J. C. Leiter, and J. A. Daubenspeck, "Reduced respiratory-related evoked activity in subjects with obstructive sleep apnea syndrome," J. Appl. Physiol., vol. 94, pp. 429-438, 2003.

[11] R. L. Horner, J. A. Innes, K. Murphy, and A. Guz, "Evidence for reflex upper airway dilator muscle activation by sudden negative pressure in man," J. Physiol. (London), vol. 436, pp. 15-29, 1991.

[12] E. A. Doble, J. C. Leiter, S. L. Knuth, J. A. Daubenspeck, and D. J. R. Bartlett, "A noninvasive intraoral electromyographic electrode for genioglossus muscle," J. Appl. Physiol., vol. 58, pp. 1378-1382, 1985.

[13] A. Merlo, D. Farina, and R. Merletti, "A fast and reliable technique for muscle activity detection from surface EMG signals," IEEE Trans. Biomed. Eng., vol. 50, no. 3, pp. 316-323, Mar. 2003.

[14] C. Li, C. Zheng, and C. Tai, "Detection of ECG characteristic points using wavelet transforms," IEEE Trans. Biomed. Eng., vol. 42, no. 1, pp. 21-28, Jan. 1995.

[15] S. Kadambe, R. Murray, and G. F. Boudreaux-Bartels, "Wavelet transform-based QRS complex detector," IEEE Trans. Biomed. Eng., vol. 46, no. 7, pp. 838-848, Jul. 1999.

[16] S. Kadambe and G. F. Boudreaux-Bartels, "Application of the wavelet transform for pitch detection of speech signals," IEEE Trans. Inf. Theory, vol. 38, pp. 917-924, 31992.

[17] M. Khalil and J. Duchene, "Uterine EMG analysis: a dynamic approach for change detection and classification," IEEE Trans. Biomed. Eng., vol. 46, no. 6, pp. 748-756, Jun. 2000.

[18] K. Berkner and R. O. Wells, "A new hierarchical scheme for approximating the continuous wavelet transform with applications to edge detection," IEEE Signal Process. Lett., vol. 6, no. Aug., pp. 193-195, 1999.

[19] S. Meignen, P. Y. Gumery, and H. Roux-Buisson, "Contribution au shéma hiérarchique de Berkner pour l'approximation de la transformée en ondelettes: application à la mesure électromyographique du temps de latence du muscle génioglosse," Traitement du Signal, vol. 20, no. 4, pp. 375-388, 2003.

[20] P. Y. Guméry, S. Meignen, H. Roux-Buisson, E. Aithocine, and P. Lévy, "Reconstruction process of the Berkner transform: application to scale range determination in a genioglossus EMG reflex time-scale detector," in Proc. 25th Аnnu. IEEE-EMBS Conf, Cancun, Mexico, Sep. 17-21, 2003, pp. 2606-2609.

[21] N. S. Arikidis, E. W. Abel, and A. Foster, "Interscale wavelet maximum-a fine to coarse algorithm for wavelet analysis of the EMG interference pattern," IEEE Trans. Biomed. Eng., vol. 49, no. 4, pp. 337-344, Apr. 2002.

[22] L. Lo Conte, R. Merletti, and G. V. Sandri, "Hermite expansion of compact support waveforms: applications to myoelectric signals," IEEE Trans. Biomed. Eng., vol. 41, no. 12, pp. 1147-1159, Dec. 1994.

[23] J. R. Wheatley, W. S. Mezzanotte, D. J. Tangel, and D. P. White, "Influence of sleep on genioglossus muscle activation by negative pressure in normal men," Am. Rev. Respir. Dis., vol. 148, pp. 597-605, 1993.

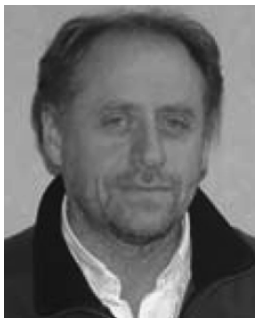

Pierre Yves Guméry received the Ph.D. degree in biomedical engineering from Joseph Fourier University, Grenoble, France, in 1992.

Since 1993, he has been an Associate Professor with Joseph Fourier University. He conducts research in the area of physiology system modeling and biosignal processing at the Laboratory TIMC-PRETA (UMR CNRS 5525). He is also Head of the Master of Models and Instruments in Medecine and Biology of the same university

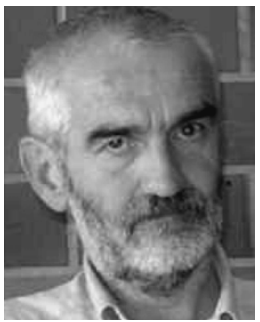

Hervé Roux-Buisson received the Doctorat d'Etat in solid-state physics from Joseph Fourier University, Grenoble, France, in 1980.

$\mathrm{He}$ worked on solar energy in Ivory Coast and on solid-state physics in Grenoble. Currently, his research interests are biosignal processing and system modeling. He is an Assistant Professor of physics and electronics.

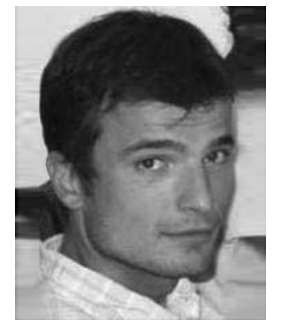

Sylvain Meignen received the M.S. degree from the INPG university, Grenoble, France, in 1998 and the Ph.D. degree from Joseph Fourier Univeristy, Grenoble, in 2001.

At present, he is an Assistant Professor with the "Ecole Nationale Supérieure d'Informatique et de Mathématiques Appliquées de Grenoble." His research interests are in the fields of biosignal processing, image processing, and texture analysis by means of wavelets and multiresolution approaches.

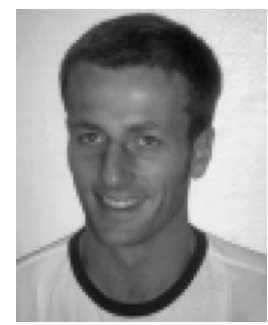

François Louis Comyn recieved the D.E.A. degree in biology and physiology of respiration and circulation from the Paris University, France, in 2001.

He studied the genioglossus response to pressure drop stimulation. Currently, he is a dental surgeon.

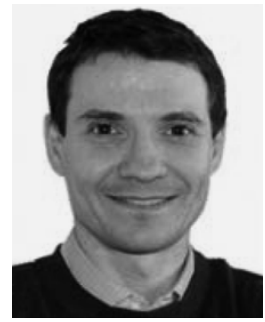

Maurice Dematteis received the M.D. degree in 1996 and the Ph.D. degree in neuroscience in 2001 , both from Joseph Fourier University,Grenoble.

In 2003, he became an Assistant Professor of physiology, Grenoble University Hospital, and a Faculty member of Medicine. His research focuses on the study of peripheral nervous system involvement in sleep-disordered breathing.

Bernard Wuyam received the M.D. degree in 1988 and the Ph.D. degree in physiology in 1991 from Joseph Fourier University, Grenoble, France. He was subsequently trained at Charing Cross \& Westminster Medical School (Pr Abe GUZ), London, U.K.

Since then, he worked in the field of lung function testing and the control of breathing. He is currently working in the Universitary Hospital of Grenoble and is a member of the Laboratory HP2-INSERM.

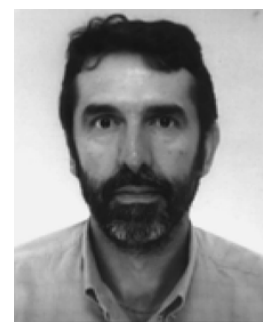

Jean Louis Pépin received the M.D. degree in 1989 from the University of Monpellier, Monpellier, France, and the Ph.D. degree in clinical physiology in 2001 from Joseph Fourier University, Grenoble, France.

$\mathrm{He}$ focuses on sleep-breathing disorders and chronic respiratory failures and their treatment. He is a Professor of clinical physiology at Joseph Fourier University.

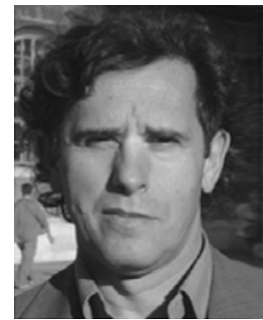

Patrick Lévy received the M.D. degree in 1981 and the Ph.D. degree in biology in 1989, both from Joseph Fourier University, Grenoble, France.

$\mathrm{He}$ had continuous interest in clinical research in the sleep-disordered breathing area and focused on the effects of chronic and intermittent hypoxia. He is a Professor of physiology, Head of Pulmonary Function Test Unit and the Sleep Laboratory in the Grenoble University Hospital. 